\title{
Pentraxin 3 regulates neutrophil infiltration to the brain
}

\section{during neuroinflammation [version 1; peer review: 2}

\section{approved]}

\author{
Ivana Rajkovic, Raymond Wong, Eloise Lemarchand, Rory Tinker(iD, \\ Stuart M. Allan, Emmanuel Pinteaux (D)
}

Faculty of Biology, Medicine and Health, University of Manchester, Manchester, UK

V1 First published: 15 May 2019, 1:10
https://doi.org/10.12688/amrcopenres.12875.1

Latest published: 15 May 2019, 1:10

https://doi.org/10.12688/amrcopenres.12875.1

Open Peer Review
Approval Status
Morsion 1
Argel Aguilar Valles, Carleton University,
Ottawa, Canada
2. Sébastien Jaillon, Humanitas University,
Milan, Italy
Any reports and responses or comments on the
article can be found at the end of the article.

\section{Abstract}

Introduction: The acute phase protein pentraxin 3 (PTX3) is known for its anti-inflammatory effects through downregulating neutrophil transmigration during peripheral inflammation. Furthermore, we have previously demonstrated a neuroprotective and neuroreparative effect of PTX3 after cerebral ischaemia. Here we investigated, to our knowledge for the first time, the role of PTX3 in neutrophil transmigration and neurotoxicity following lipopolysaccharide (LPS)induced cerebral inflammation and cerebral ischaemia.

Methods: Neutrophil transmigration through interleukin-1 $\beta$ (IL-1 $\beta$ ) activated brain endothelium and neurotoxicity of neutrophils isolated from wild-type (WT) or PTX3 knock-out (KO) mice was assessed in vitro. Primary cortical neuronal death after treatment with transmigrated neutrophils was quantified by lactate dehydrogenase (LDH) assay. Cerebral inflammation or ischemia was induced in WT and PTX3 KO mice via intrastriatal LPS injection or by transient middle cerebral artery occlusion (MCAo) respectively. Subsequent neutrophil infiltration in the brain was assessed by immunohistochemistry and the expression of pro-inflammatory cytokines interleukin-6 (IL-6) and IL-1 $\beta$ by enzyme-linked immunosorbent assay (ELISA).

Results: Neutrophils isolated from WT mice after intrastriatal LPS injection transmigrated significantly more through IL-1 $\beta$ activated brain endothelium compared to neutrophils from PTX3 KO mice. Transmigrated WT and PTX3 KO neutrophils were significantly more neurotoxic than corresponding non-transmigrated neutrophils; however, no significant differences in neurotoxicity between genotypes were observed. PTX3 reduced the number of transmigrated neutrophils to the brain after intrastriatal LPS injection. Furthermore, PTX3 KO mice showed significantly increased levels of neutrophils in the brain after LPS administration or in the ischaemic hemisphere after MCAo, compared to WT mice.

Conclusion: Our study shows that PTX3 regulates neutrophil 
transmigration in the CNS during neuroinflammation, demonstrating the potential of PTX3 as an effective therapeutic target in neuroinflammatory conditions.

\section{Keywords}

Pentraxin-3, inflammation, neutrophil infiltration, neurotoxicity, neuroprotection

\section{Stroke This article is included in the Stroke Association Assciation gateway.}

Corresponding author: Emmanuel Pinteaux (emmanuel.pinteaux@manchester.ac.uk)

Author roles: Rajkovic I: Data Curation, Formal Analysis, Investigation, Methodology, Validation, Writing - Review \& Editing; Wong R: Methodology, Validation, Writing - Review \& Editing; Lemarchand E: Data Curation, Formal Analysis, Methodology; Tinker R: Data Curation, Formal Analysis, Writing - Review \& Editing; Allan SM: Conceptualization, Funding Acquisition, Project Administration, Validation, Writing - Review \& Editing; Pinteaux E: Conceptualization, Funding Acquisition, Project Administration, Supervision, Validation, Writing - Original Draft Preparation, Writing - Review \& Editing

Competing interests: No competing interests were disclosed.

Grant information: This work was supported by funding from the British Heart Foundation (Reference: FS/14/70/31006), the Stroke Association (Reference: TSA2017/03) and the Medical Research Council (Reference: MR/N003586/10).

The funders had no role in study design, data collection and analysis, decision to publish, or preparation of the manuscript.

Copyright: ( 2019 Rajkovic I et al. This is an open access article distributed under the terms of the Creative Commons Attribution License, which permits unrestricted use, distribution, and reproduction in any medium, provided the original work is properly cited.

How to cite this article: Rajkovic I, Wong R, Lemarchand E et al. Pentraxin 3 regulates neutrophil infiltration to the brain during neuroinflammation [version 1; peer review: 2 approved] AMRC Open Research 2019, 1:10

https://doi.org/10.12688/amrcopenres.12875.1

First published: 15 May 2019, 1:10 https://doi.org/10.12688/amrcopenres.12875.1 


\section{Introduction}

Neuroinflammation triggered from an initial brain insult such as acute brain injury, stroke or infection is characterized by a potent central and peripheral inflammatory response that is known to activate local resident brain cells as well as peripheral immune cells, such as neutrophils, which then infiltrate into the lesion and release inflammatory mediators, exacerbating neuronal death and brain injury (Kim et al., 2016). There is extensive evidence demonstrating that neutrophils mediate disruption of the blood-brain barrier (BBB), oedema, and brain damage after ischaemic stroke, through release of reactive oxygen species (ROS), proteases and cytokines (Jickling et al., 2015). Thus, targeting neutrophils as a potential stroke treatment is an attractive therapeutic option.

Pentraxin-3 (PTX3) is an acute-phase protein known to exert antiinflammatory and protective effects in peripheral inflammatory conditions including infection, acute myocardial infarction and lung inflammation (see (Erreni et al., 2017) for review). Indeed, PTX3 knock-out (KO) mice demonstrated greater myocardial injury, which was associated with greater neutrophil infiltration when compared to wild type (WT) mice (Salio et al., 2008). Furthermore, PTX3 has been shown to reduce neutrophil transmigration in a P-selectin-dependent manner during lung inflammation (Deban et al., 2010). Moreover, a previous published study indicated that PTX3 is stored in neutrophil granules, localised in neutrophil extracellular traps (NETs) (Jaillon et al., 2007), which could contribute to the regulation of neutrophil infiltration.

Our previous studies in stroke have demonstrated that PTX3 expressed in the brain after experimental cerebral ischemia (induced by occlusion of the middle cerebral artery (MCAo)) plays a critical role in the restoration of blood flow (Rajkovic et al., 2018) and integrity of the BBB (Rodriguez-Grande et al., 2014), and is a key regulator of long-term angiogenesis, neurogenesis and neuroprotection (Rajkovic et al., 2018). Despite these previous observations, the mechanisms by which PTX3 regulates neuroinflammation remains largely undefined, and the role of PTX3 of neutrophil transmigration in the brain during CNS inflammation remains completely unknown. This study aimed to test the hypothesis that PTX3 regulates neutrophil transmigration during neuroinflammation. Here we report, to our knowledge for the first time, that PTX3 regulates neutrophil transmigration in vitro and decreases neutrophil recruitment to the brain after intrastriatal lipopolysaccharide (LPS) injection and after cerebral ischaemia. In addition, neutrophil infiltration triggered neurotoxicity; however, no significant differences in neurotoxicity between genotypes was observed. Collectively, our findings suggest that PTX3 may be an effective potential therapeutic target, as it may prevent ischaemic damage through reductions in neutrophil recruitment to the brain after cerebral ischaemia.

\section{Methods}

\section{Animals}

Male PTX3 KO mice and WT littermates (total $n=21$ and $n=22$, respectively) were obtained by in-house breeding from heterozygote mice (bred on C57/BL6 background) (obtained from
Dr. Cecilia Garlanda, Humanitas Clinical and Research Center, Rozzano, Italy), and were genotyped as described previously (Rodriguez-Grande et al., 2014). Experiments were carried out on weight-matched 12-14-week-old WT or PTX3 KO males. Animals were maintained under standard laboratory conditions: temperatures of $21 \pm 1^{\circ} \mathrm{C}, 55 \pm 10 \%$ humidity, $12 \mathrm{~h}$ light-dark cycle, ad libitum access to food and water. All animal procedures were conducted in agreement with the Animal Scientific Procedures Act (1986) and the European Council Directive 2010/63/EU, and were approved by the Home Office and Animal Welfare and Ethics Review Board, University of Manchester (UK). All experiments followed the ARRIVE (Kilkenny et al., 2012) guidelines. Animals were assigned to experimental groups in a random manner, and surgical procedures were conducted during daylight by an experimenter blinded to the genotype.

\section{Neutrophil isolation}

Neutrophil isolation was repeated three times in three separate experiments. The 8-week-old WT and PTX3 KO mice (one WT mouse and one PTX3 KO mouse per each neutrophil isolation) were culled via cerebral dislocation. The femur and tibia were then dissected out and maintained in Roswell Park Memorial Institute (RPMI) medium (Thermo-Fisher Scientific, $\mathrm{UK}$ ) at room temperature (RT). The bone marrow was then extracted from the femur and tibia via flushing with RPMI medium through a 25-gauge $(G)$ needle. WT and PTX3 KO suspensions were then vigorously triturated and centrifuged at $1000 \mathrm{~g}, 4^{\circ} \mathrm{C}$ for $5 \mathrm{~min}$. Subsequently, the supernatants were discarded, and $6 \mathrm{ml}$ of $0.2 \%$ saline was applied for $1 \mathrm{~min}$ and triturated (to lyse red blood cells), followed by $14 \mathrm{ml}$ of $1.2 \%$ saline (to restore osmolarity) and vigorous trituration. Suspensions were then passed through a $70-\mu \mathrm{M}$ strainer and centrifuged at $2000 \mathrm{~g}, 4^{\circ} \mathrm{C}$ for $10 \mathrm{~min}$. Supernatants were discarded and pellets were resuspended in $5 \mathrm{ml}$ HBSS (Thermo-Fisher Scientific, UK) and triturated. Suspensions were then added to $62 \%$ isotonic percoll (Sigma-Aldrich, UK) and centrifuged at $1000 \mathrm{~g}, 4^{\circ} \mathrm{C}$ for $30 \mathrm{~min}$. Excess liquid was then removed and pellets were resuspended in $10 \mathrm{ml}$ HBSS and triturated. Finally, suspensions were centrifuged at $2000 \mathrm{~g}, 4^{\circ} \mathrm{C}$ for $5 \mathrm{~min}$, supernatants discarded, and pellets resuspended in $5 \mathrm{ml}$ RPMI.

\section{bEnd5 mouse brain endothelial cell line cultures}

The immortalised mouse brain endothelial cell line bEnd5, which closely resembles primary brain endothelial cells, was obtained from Public Health England (Salisbury, UK). Cells were maintained in Dulbecco's modified Eagle medium (DMEM) (Invitrogen, UK) supplemented with $10 \%$ fetal calf serum (FCS) (PAA Laboratories, UK), $1 \%$ glutamine, $1 \mathrm{U} / \mathrm{ml}$ penicillin and $100 \mu \mathrm{g} / \mathrm{ml}$ streptomycin (P/S) in a humidified incubator at $37^{\circ} \mathrm{C}$ with $5 \% \mathrm{CO}_{2}$.

\section{Primary cortical neuronal cultures}

Embryos (day 14-15 of embryonic development) were dissected out of 12-14-week-old WT pregnant C57BL/6 female mice (Charles River, UK) following euthanasia by cervical dislocation. One pregnant mouse was used for a single experiment, which was repeated three times in three separate experiments. The cortex of each embryo was dissected out and placed into starve 
medium (DMEM with $1 \% \mathrm{P} / \mathrm{S})$ at $37^{\circ} \mathrm{C}$. Trypsin $(562.5 \mathrm{U} / \mathrm{ml})$ (Sigma Aldrich, UK) and DNase (417 U/ml) (Thermo-Fisher Scientific, UK) were then added, and suspensions were placed in a shaker at $37^{\circ} \mathrm{C}$ for $30 \mathrm{~min}$. Subsequently, FCS was added for 2 min in order to deactivate trypsin, which was then removed by three subsequent washes with wash medium (DMEM, 1\% P/S, 10\% FCS). Brains were then resuspended in $5 \mathrm{ml}$ seeding media (Neurobasal Medium (NBM) (ThermoFisher Scientific, UK), 5\% Plasma Derived Serum (PDS) (First Link Ltd, UK), 1\% P/S, $1 \%$ glutamine (Sigma-Aldrich, UK), 2\% B27 supplement with antioxidants (Thermo-Fisher Scientific, UK)) and triturated. The suspension was then passed through an $80-\mu \mathrm{M}$ nylon filter. To prevent glial proliferation, 5-fluoro-2deoxyuridine (30 $\mu \mathrm{M}$, Sigma-Aldrich, UK) was added. Cells were counted and seeded at $1.8 \times 10^{5} \mathrm{cells} / \mathrm{cm}^{2}$ on to Poly-DLysine-coated 24- or 96-well plates. On day in vitro (DIV) 5 medium was replaced with change medium (NBM, 5\% PDS, $1 \%$ $\mathrm{P} / \mathrm{S}, 1 \%$ glutamine, and 2\% B27 supplement without antioxidants), followed by half medium change every 2 days until DIV 12-13.

\section{Neutrophil transmigration assay}

Neutrophil transmigration was carried out as described previously (Allen et al., 2012). Briefly, 1 x $10^{5}$ cells/well of bEnd5 cells were seeded onto Transwell inserts $(6.5 \mathrm{~mm}$ with $3.0 \mu \mathrm{m}$ pore polycarbonate membrane (Sigma-Aldrich, UK)) for $24 \mathrm{~h}$. bEnd5 cells were then pre-treated with recombinant mouse interleukin (IL)-1 $\beta$ (100 ng/ml) (R\&D Systems, UK) or vehicle (low $0.1 \%$ endotoxin bovine serum albumin (BSA) in $\mathrm{NaCl}$ ) for $4 \mathrm{~h}$. Subsequently, $2 \times 10^{5}$ neutrophils isolated from WT or PTX3 KO mice were added to the luminal (top) compartment of vehicle (WT neutrophils) or IL-1 $\beta$ (WT or PTX3 KO neutrophils) treated inserts and allowed to transmigrate for $24 \mathrm{~h}$. Neutrophils were collected from the abluminal compartments, centrifuged at $400 \mathrm{~g}$ for $10 \mathrm{~min}$ and counted with a hemocytometer. Neutrophil transmigration was represented as fold increase compared to vehicle-treated cultures.

\section{Neutrophil-mediated neurotoxicity}

Neuronal cultures grown on to 96-well plates were incubated with WT or PTX3 KO neutrophil transmigrated conditioned media or WT or PTX3 KO non-transmigrated neutrophil conditioned media (containing $4 \times 10^{5}$ cells $/ \mathrm{ml}$ ) for $20 \mathrm{~h}$. A lactate dehydrogenase $(\mathrm{LDH})$ cell death assay was then carried out to quantify neuronal cell death in cultures.

\section{Intrastriatal lipopolysaccharide (LPS) injection}

Cerebral inflammation was achieved by intrastriatal bacterial lipopolysaccharide (LPS) injection as described previously (Giles et al., 2015). Briefly, 12-14-week-old WT or PTX3 KO male mice (five mice per experimental group for the 4-h time point; five WT and seven PTX3 KO mice for the 24-h time point) were placed in a stereotaxic frame and anaesthetised with $4 \%$ isoflurane $(30 \%$ oxygen and $70 \%$ nitrous oxide gas, AbbVie Ltd, UK), followed by maintenance at $1.75 \%$. A craniotomy was performed, and mice were injected intracerebrally using a glass microneedle with $4 \mu \mathrm{g}$ LPS (Sigma-Aldrich, UK) or vehicle $(9 \% \mathrm{NaCl})$ into the following co-ordinates from bregma: anteriorposterior $-0.0 \mathrm{~mm}$, lateral $-2.0 \mathrm{~mm}$, ventral $-2.5 \mathrm{~mm}$. Rate $=$ $0.5 \mu \mathrm{l} / \mathrm{min}$ ). Animals recovered for $4 \mathrm{~h}$ or $24 \mathrm{~h}$, following which collection of cardiac blood and tissue processing was performed as described below.

\section{Induction of cerebral ischaemia by transient middle} cerebral artery occlusion (MCAo)

12-14-week-old WT or PTX3 KO male mice (6 mice per experimental group) were subjected to experimental cerebral ischemia induced by occlusion of the middle cerebral artery (MCAo) for $15 \mathrm{~min}$ followed by $48 \mathrm{~h}$ reperfusion and recovery as described previously (Rajkovic et al., 2018). Animals that did not demonstrate a minimum of $70 \%$ decrease in CBF from pre-occlusion baseline were eliminated from the study.

\section{Tissues processing}

Animals ( $\mathrm{n}=18$ PTX3 KO and $\mathrm{n}=16$ WT mice) were euthanised with $4 \%$ isoflurane, and cardiac blood was removed from the right ventricle and centrifuged at $2000 \mathrm{~g}, 4^{\circ} \mathrm{C}$ for $10 \mathrm{~min}$, in order to obtain plasma from blood. Mice were transcardially perfused with $0.9 \%$ saline followed by $4 \%$ paraformaldehyde (PFA) (Sigma-Aldrich, UK). Brains were then removed, post-fixed in $4 \%$ PFA for $24 \mathrm{~h}$ then in $30 \%$ sucrose for a further $24 \mathrm{~h}$, and were serially sectioned into $30-\mu \mathrm{m}$ sections on a freezing sledge microtome (Bright, Cambridgeshire, UK). Cryoprotectant solution (30\% ethylene glycol, $20 \%$ glycerol, $0.66 \%$ sodium phosphate dibasic dehydrate, $0.079 \%$ sodium dihydrogen orthophosphate 1-hydrate, in distilled water $\left.\left(\mathrm{dH}_{2} \mathrm{O}\right)\right)$ was used for long term storage of sections at $-20^{\circ} \mathrm{C}$.

\section{Immunohistochemistry}

Free-floating brain sections were washed three times with phosphate buffered saline (PBS) and then incubated with blocking buffer (10\% normal donkey serum (NDS) (Jackson laboratories, Bar Harbor, ME, USA), $0.3 \%$ Triton X-100 (Sigma-Aldrich, UK) and PBS) for $1 \mathrm{~h}$. After washing, sections were incubated overnight at $4{ }^{\circ} \mathrm{C}$ with rabbit anti-mouse neutrophil primary antibody (SJC, 1:10000, a gift from Dr S. J. Campbell, University of Cambridge, UK) diluted in primary antibody buffer (2\% NDS, $0.3 \%$ Triton X-100 and PBS). Subsequently, sections were washed three times with PBS, and then incubated with AlexaFluor 488-conjugated secondary antibody (1:500, Donkey antirabbit, Cat \# A-21206, RRID AB_2535792), ThermoFisher, UK) for $2 \mathrm{~h}$. Following three washes with PBS, sections were mounted in $\mathrm{dH}_{2} \mathrm{O}$ onto glass slides, and dried in the dark at RT for 24 h. Finally, slides were cover slipped with Prolong Gold with DAPI mounting solution (ThermoFisher, UK). An Olympus BX51 upright microscope (Olympus, Japan) (10 x objective) was used to collect high power field images, which were captured via a Coolsnap ES camera (photometrics, USA) through MetaVue software (Molecular Devices, USA). Coronal sections of the same co-ordinates (approximately bregma level $0.84 \mathrm{~mm}$, according to the Mouse Brain Atlas) were used for analyses of immunohistochemistry micrographs. ImageJ software (version 1.50i, National Institutes of Health, USA) was used to manually count the number of neutrophils $/ \mathrm{mm}^{2}$ in contralateral and ipsilateral (LPS injected or ischaemic) hemispheres in three randomly selected regions in the striatum and cortex (intrastriatal LPS injection study) and penumbra (48 h stroke study) regions. All images were collected and analysed by a blinded experimenter. 


\section{Tissue homogenates preparation}

Liver and spleen were isolated and homogenised using a mechanical homogeniser (IKA, USA) in homogenisation buffer (5 $\mu \mathrm{l} / \mathrm{mg}$ of tissue) (50 mM Tris-HCL, $150 \mathrm{mM} \mathrm{NaCl}, 5 \mathrm{mM}$ $\mathrm{CaCl}_{2}, 0.02 \% \mathrm{NaN}_{3}, 1 \%$ Triton $\mathrm{X}-100$, protease inhibitor cocktail 1 and phosphatase inhibitors $(1 \mathrm{mM}$ sodium orthovanadate and $5 \mathrm{mM}$ sodium fluoride)). Samples were then incubated on ice for $30 \mathrm{~min}$, and then centrifuged at $14000 \mathrm{rpm}$ for $30 \mathrm{~min}$ at $4^{\circ} \mathrm{C}$, after which supernatants were stored at $-80^{\circ} \mathrm{C}$.

\section{Enzyme linked immunosorbent assay (ELISA)}

An ELISA (R\&D Systems, UK, Cat \# DY406-05 and DY401$05)$ was used to detect IL-6 and IL-1 $\beta$ ( $\mathrm{pg} / \mathrm{ml}$ ) levels of plasma, liver and spleen samples from WT and PTX3 KO mice (after LPS administration or MCAo) following the manufacturer's instructions. Optical densities were read in a plate reader at $450 \mathrm{~nm}$ corrected for baseline at $570 \mathrm{~nm}$ (Synergy HT plate reader, BioTek). Concentrations of the samples were obtained by interpolating from a standard curve fitted by sigmoidal APL equation, and were expressed as $\mathrm{pg} / \mathrm{ml}$. The detection limit of the assays was $23 \mathrm{pg} / \mathrm{ml}$ for IL-6 and $16 \mathrm{pg} / \mathrm{ml}$ for IL- $1 \beta$.

\section{Statistical analyses}

All data are presented as mean values \pm standard error of the mean (SEM) $(n=3-5$ in vitro experiments; $n=5-7$ in vivo experiments, based on previous published data (Rajkovic et al., 2018; Rodriguez-Grande et al., 2014). The normality of data was assessed with the Shapiro-wilk test, and appropriate transformations were applied when necessary. Statistical analyses performed on normally distributed data were unpaired student's t-test (comparison of two groups), ordinary or repeated measures (where appropriate) one-way analysis of variance (ANOVA) followed by Dunnett's corrected post-hoc analysis (comparison of multiple groups with a control group) or Bonferroni multiple comparisons post-hoc test (for comparisons between multiple groups), and repeated measures two-way ANOVA test with Sidak corrected post hoc (multiple comparisons between matched hemisphere and genotype groups). The Mann-Whitney test was used for data that were not normally distributed. $\mathrm{P}<0.05$ was considered to indicate a statistically significant difference. Statistical analyses were carried out using GraphPad Prism 7.0.

\section{Results \\ PTX3 regulates neutrophil transmigration and neuroprotection}

Here we sought to investigate the role of PTX3 in regulation of neutrophil transmigration in an in vitro model of neutrophil transmigration through IL- $1 \beta$ activated brain endothelium. The number of bone marrow-derived neutrophils prepared from WT or PTX3 KO mice did not differ under normal conditions (Figure 1Ai). Neutrophils isolated from WT and PTX3 KO mice transmigrated significantly more through IL-1 $\beta$ activated brain endothelium, compared to WT neutrophils through vehicle treated endothelium (WT 2.4-fold, p<0.001; PTX3 KO 0.7-fold, $\mathrm{p}<0.05$ ). However, neutrophils isolated from PTX3 KO mice transmigrated significantly less $(1.74$-fold, $\mathrm{p}<0.001)$ than WT isolated neutrophils through the IL-1 $\beta$ activated brain endothelium (Figure 1Aii).
We next determined the effect of WT and PTX3 KO transmigrated neutrophils on neuronal viability. Neurones cultured with WT or PTX3 KO transmigrated neutrophils for $24 \mathrm{~h}$ showed a significantly greater cell death (WT 3.4-fold, $\mathrm{p}<0.01$; PTX3 KO 1.8-fold, $\mathrm{p}<0.05)$ compared to neurones exposed to naïve WT or PTX3 KO neutrophils. However, we did not find a significant difference in neurotoxicity between transmigrated WT and transmigrated PTX3 KO neutrophils (Figure 1Aiii). These data suggest that PTX3 did not affect neutrophil transmigrationinduced neurotoxicity.

We repeated the study under inflammatory conditions, isolating neutrophils from WT and PTX3 KO mice after intrastriatal LPS injection (Figure 1B). Contrary to our findings under normal conditions, we noted a significant reduction (1.4-fold, $\mathrm{p}<0.01)$ in the number of neutrophils isolated from the bone marrow of PTX3 KO mice compared to WT mice (Figure 1Bi). Similarly, WT and PTX3 KO isolated neutrophils following LPS injection showed significantly greater transmigration through IL-1 $\beta$ activated brain endothelium, compared to WT neutrophils through vehicle-treated endothelium (WT 2.8-fold, $\mathrm{p}<0.001$; PTX3 KO 1.3-fold, $\mathrm{p}<0.01$ ). Furthermore, transmigration was significantly reduced $(1.5$-fold, $\mathrm{p}<0.01)$ in PTX3 KO isolated neutrophils compared to WT isolated neutrophils, through the IL-1 $\beta$ activated brain endothelium (Figure 1Bii). Our study also demonstrated a significant increase in percentage of neuronal death (WT 1.9-fold, p<0.01; PTX3 KO 1.2-fold, $\mathrm{p}<0.05)$ in cultures incubated with WT or PTX3 KO transmigrated neutrophils, compared to the corresponding naïve cultures, although no significant difference between genotypes was observed (Figure 1Biii). Collectively, these findings suggest that PTX3 is an important regulator of in vitro neutrophil transmigration through the brain endothelium.

\section{PTX3 reduces neutrophil transmigration after intrastriatal LPS injection}

Neutrophil infiltration in to the brain has been reported after intrastriatal LPS injection (Giles et al., 2015). Therefore, we examined whether PTX3 regulates neutrophil infiltration to the brain during this central inflammatory paradigm. We showed a significant increase in the number of neutrophils in ipsilateral hemispheres (LPS-injected) compared to corresponding contralateral (vehicle-injected) hemispheres in WT (7-fold, $\mathrm{p}<0.05$ ) and PTX3 KO (33-fold, $\mathrm{p}<0.001)$ mice, $4 \mathrm{~h}$ after injection (Figure 2Ai and ii). PTX3 KO mice showed increased (2-fold, $\mathrm{p}<0.05$ ) amounts of neutrophils in the ipsilateral hemisphere compared to WT mice. Neutrophils localised near the ventricles in both WT and PTX3 KO mice after LPS injection, and were not observed in the striatum or cortex. In addition, no significant difference in IL-6 or IL-1 $\beta$ levels in plasma were detected between genotypes (Figure 2Aiii and iv). We also assessed neutrophil infiltration in the striatum and cortex of WT and PTX3 KO mice $24 \mathrm{~h}$ after intrastriatal LPS injection. Our data show a significant increase in the number of neutrophils in ipsilateral hemispheres compared to corresponding contralateral hemispheres of WT and PTX3 KO mice, in the striatum (WT 95-fold, $\mathrm{p}<0.05$; PTX3 KO 257-fold, $\mathrm{p}<0.001$ ) and the cortex (WT 45-fold, p<0.01; PTX3 KO 63-fold, $\mathrm{p}<0.001$ ) 
$\mathbf{A}$

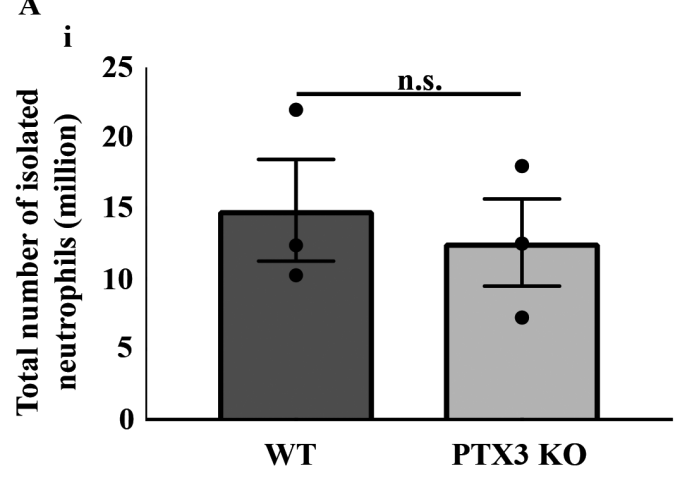

Neutrophil transmigration

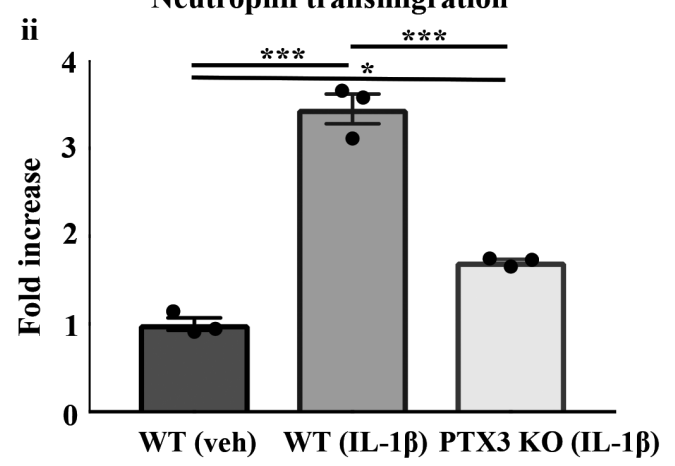

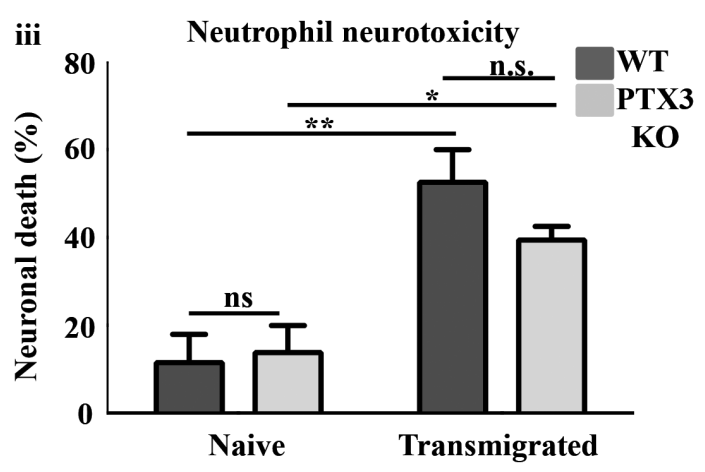
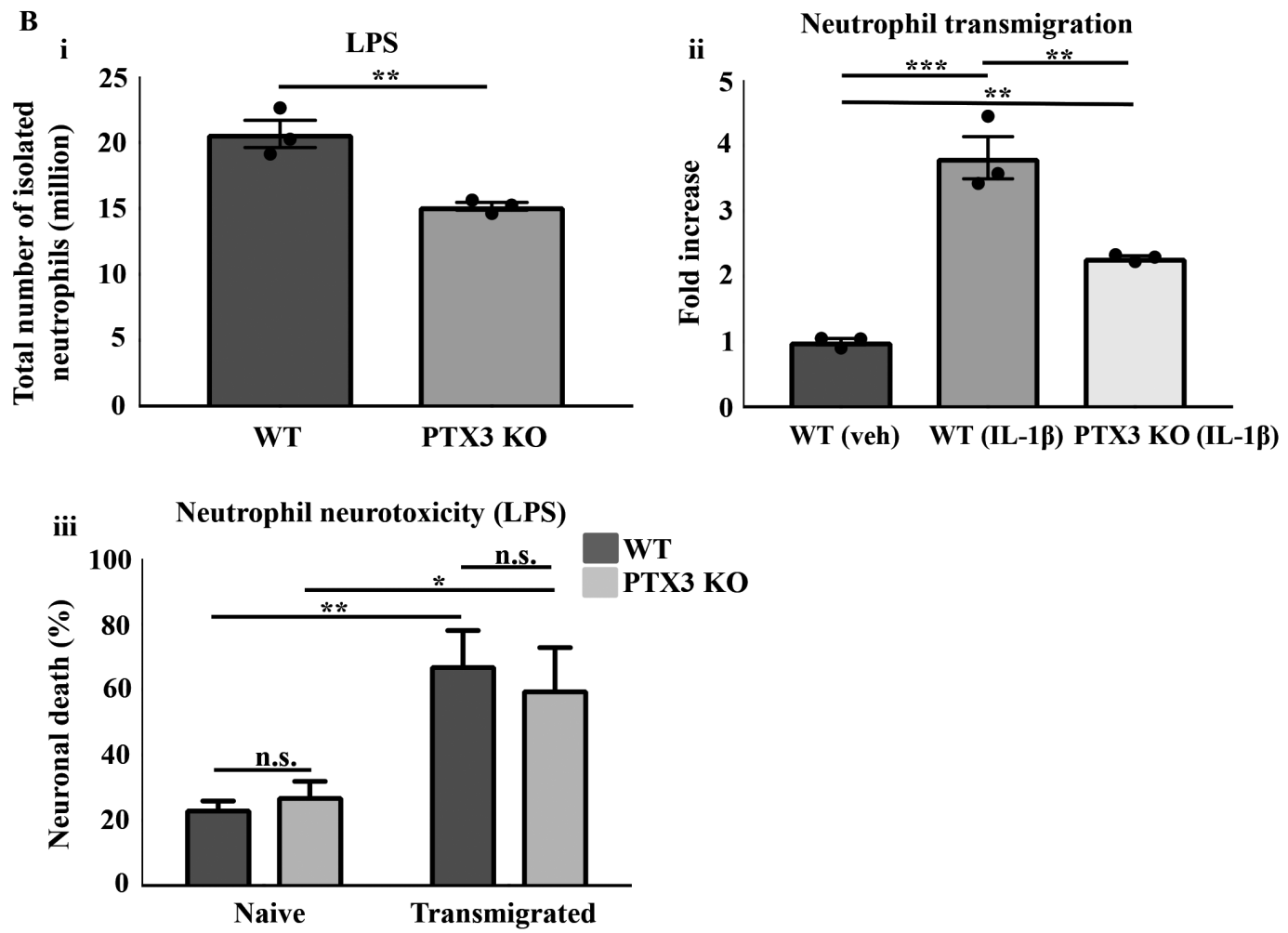

Figure 1. PTX3 promotes neutrophil transmigration in vitro. (Ai, Bi) Total number of neutrophils isolated, (Aii, Bii) neutrophil transmigration, (Aiii, Biii) and neutrophil-mediated neurotoxicity under normal and intrastriatal LPS brain injected conditions in WT and PTX3 KO mice, respectively. The total number of neutrophils and neutrophil transmigration in WT and PTX3 KO mice were quantified by cell counting with a cell haemocytometer ( $\mathbf{A i}$, Aii, Bi, Bii). Neutrophil mediated neurotoxicity was quantified by LDH assay (Aiii, Biii). Statistical analyses performed using unpaired Student's t-test (ns $\left.\mathrm{P}>0.05,{ }^{* *} \mathrm{P}<0.01\right)(\mathbf{A} \mathbf{i}, \mathbf{B} \mathbf{i})$, one-way ANOVA followed by Bonferroni multiple comparisons post-hoc test $\left({ }^{\star} \mathrm{P}<0.05,{ }^{* \star} \mathrm{P}<0.01\right.$, ${ }^{\star \star *} \mathrm{P}<0.001$ ) (Aii, Bii), two-way ANOVA followed by Sidak corrected post-hoc analysis (ns $\mathrm{P}>0.05$, $\left.{ }^{*} \mathrm{P}<0.05,{ }^{* *} \mathrm{P}<0.01\right)(\mathbf{A i i i}, \mathbf{B i i i})$. All data expressed as mean $\pm \mathrm{SEM}(\mathrm{n}=3)(\mathbf{A i}-\mathbf{i i i}, \mathbf{B})$. 

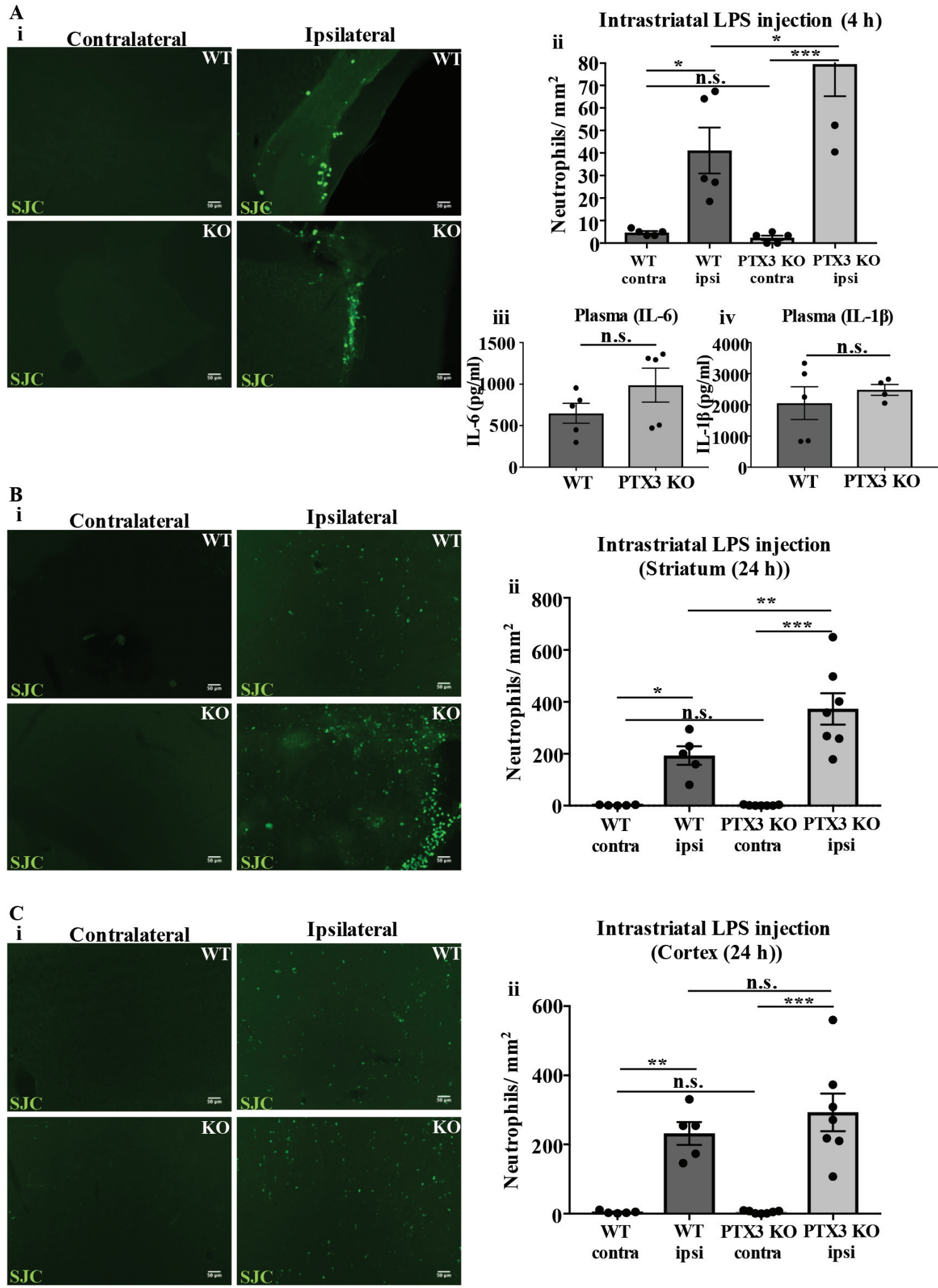

Figure 2. PTX3 limits neutrophil infiltration in the brain after intrastriatal LPS injection. (Ai, Bi, Ci) Neutrophils (green) labelled with SJC antibody in the brain of WT and PTX3 KO mice $4 \mathrm{~h}$ and $24 \mathrm{~h}$ after intrastriatal LPS injection. Scale bar, $50 \mu \mathrm{M}$. (A ii, B ii, C ii) Number of neutrophils per $\mathrm{mm}^{2}$ were counted manually with ImageJ software. (A iii, A iv) Levels (pg/ml) of pro-inflammatory cytokines IL-6 and IL-1 3 in plasma of WT and PTX3 KO mice $4 \mathrm{~h}$ after intrastriatal LPS injection were detected by ELISA. Statistical analyses were assessed by repeated measures two-way ANOVA followed by Sidak corrected post-hoc analysis ( $\mathrm{ns} P>0.05$, ${ }^{*} \mathrm{P}<0.05$, ${ }^{* *} \mathrm{P}<0.01$, $\left.{ }^{* * *} \mathrm{P}<0.001\right)(\mathbf{A i i}, \mathbf{B i i}, \mathbf{C i i})$, or unpaired Student's t-test ( $\mathrm{ns} P>0.05)($ Aiii, Aiv). All data expressed as mean \pm SEM $(n=5-7)(\mathbf{A}-\mathbf{C})$. 
(Figure $2 \mathrm{~B}, \mathrm{C}$ ). Similar to our observation at $4 \mathrm{~h}$, PTX3 KO mice showed $(2$-fold, $\mathrm{p}<0.01)$ increased numbers of neutrophils in the ipsilateral striatum compared to WT mice (Figure 2B). In contrast, we did not observe a significant difference in the number of neutrophils in the ipsilateral hemisphere between genotypes in the cortex (Figure 2C). In summary, our study demonstrates a novel role of PTX3 as a regulator of neutrophil trafficking to the brain in response to central inflammatory challenge.

PTX3 KO mice demonstrate greater neutrophil infiltration in the brain $48 \mathrm{~h}$ after cerebral ischaemia

Consequently, we examined neutrophil transmigration to the brain $48 \mathrm{~h}$ after cerebral ischaemia. WT and PTX3 KO mice demonstrated a significant increase (WT 38-fold, $\mathrm{p}<0.001$; PTX3 KO 197-fold, $\mathrm{p}<0.001$ ) in the number of neutrophils in the ipsilateral hemisphere compared to corresponding contralateral hemisphere (Figure 3A). Increased (2-fold, $\mathrm{p}<0.001$ ) levels of neutrophils in the PTX3 KO ipsilateral hemisphere compared to WT mice were also observed (Figure 3A). We also assessed expression of pro-inflammatory cytokines IL-1 $\beta$ and IL-6 in the liver and spleen $48 \mathrm{~h}$ after MCAo (Figure $3 \mathrm{~B}-\mathrm{E}$ ). Our data show a significant increase $(2$-fold, $\mathrm{p}<0.05)$ in IL-1 $\beta$ expression in the liver of PTX3 KO mice compared to WT mice (Figure 3B). Levels of IL-1 $\beta$ and IL- 6 in the plasma were below the limit of detection, and we found no significant differences between genotypes in the spleen, or the liver (IL-6) (Figure 3C, E). These findings suggest that PTX3 prevents neutrophil migration to the brain after cerebral ischaemia without affecting peripheral circulating cytokine levels.

\section{Discussion}

The acute-phase protein PTX3 has been extensively described as a biomarker in major inflammatory disorders such as stroke, cancer or cardiovascular disease (Bonacina et al., 2013; Ryu et al., 2012; Shindo et al., 2014), and several studies indicate that PTX3 regulates neutrophil transmigration during peripheral inflammation via interaction with P-selectin (a key cell surface adhesion molecule critical for neutrophil recruitment) (Deban et al., 2010; Salio et al., 2008). However, the role of PTX3 in neutrophil infiltration during CNS inflammation remains largely unknown. Here we demonstrate for the first time that PTX3 is a key regulator of neutrophil transmigration in the CNS, but with opposing effects in vitro and in vivo. Furthermore, we demonstrate that neurotoxicity upon neutrophil transmigration is totally independent of neutrophil-derived PTX3.

Previous research has shown that PTX3 prevents excessive neutrophil infiltration, by limiting the number of infiltrated neutrophils in peripheral inflammatory conditions (Deban et al., 2010; Salio et al., 2008). First, we found that PTX3 aided neutrophil transmigration in an in vitro model of IL-1 $\beta$ activated brain endothelium, since neutrophils from PTX3 KO mice showed decreased ability to transmigrate compared to WT mice, and this effect was similar using neutrophils obtained from WT and PTX3 KO mice subjected to intrastriatal injection of LPS. However, our in vivo experiments are in agreement with previously published studies on cardiac ischaemia and pleural inflammation (Deban et al., 2010; Salio et al., 2008), in that PTX3 dampens down neutrophil transmigration in the brain during neuroinflammation. Indeed, we found that intrastriatal LPS injection led to a marked decrease in neutrophil infiltration to the brain, in WT mice. However, PTX3 KO mice had significantly increased neutrophil infiltration compared to WT mice, whilst levels of circulating IL-6 and IL-1 $\beta$ were similar between WT and PTX3 KO mice, suggesting that the differential effect of LPS on neutrophil infiltration might occur through central, rather than peripheral, inflammatory mechanisms. The inhibitory effect of PTX3 on neutrophil infiltration observed after LPS challenge was also observed after cerebral ischaemia, since PTX3 KO mice had significantly increased neutrophil infiltration compared to WT mice $48 \mathrm{~h}$ after MCAo. In this condition, levels of IL-1 $\beta$ in the spleen were also significantly increased after MCAo in PTX3 KO mice, raising the possibility that in stroke neutrophil infiltration regulated by PTX3 could involve IL-1 $\beta$.

Interestingly, in our in vivo LPS study, infiltrated neutrophils were located near the ventricles $4 \mathrm{~h}$ after LPS, whereas at $24 \mathrm{~h}$ they were located in the striatum and cortex. Until recently neutrophils were thought to transmigrate across the BBB via microvessels into the perivascular parenchyma, and then enter the brain parenchyma (Takeshita \& Ransohoff, 2012). Emerging evidence indicates that there is a population of neutrophils entering the CNS through the choroid plexus (CP), which is located in third and fourth ventricles of the brain (Meeker et al., 2012; Schwartz \& Baruch, 2014). The population of neutrophils located solely by the ventricles at $4 \mathrm{~h}$ post LPS administration may be this population of neutrophils that have trafficked through the CP. Furthermore, we observed a significant reduction in total number of bone-marrow-isolated neutrophils from PTX3 KO mice compared to WT mice in our in vitro assay, which correlates with the increased number of neutrophils observed in the brains of PTX3 $\mathrm{KO}$ mice compared to WT mice after intrastriatal LPS injection, and may be indicative of more neutrophils trafficking from the bone marrow to the CNS in PTX3 KO mice than WT mice after inflammatory stimulation.

We have previously demonstrated that transmigrated neutrophils through an IL-1-activated brain endothelium acquired a neurotoxic phenotype in vitro (Allen et al., 2012), and our very recent studies found that genetic inhibition of IL-1 signalling in brain endothelial cells completely abrogated neutrophil infiltration and subsequent ischemic brain damage (Wong et al., 2019). Taking into consideration that PTX3 is readily stored in NETs (Jaillon et al., 2007), we investigated the effect of PTX3 on transmigrated neutrophil mediated neurotoxicity. Consistent with our previous study, we found that transmigrated neutrophils acquired a potent neurotoxic phenotype compared to naïve (non-transmigrated neutrophils) but found that this acquired phenotype was not significantly different between WT or PTX3 KO transmigrated neutrophils. This observation may correlate with lack of difference on acute or delayed ischemic brain damage between WT and PTX3 KO previously reported (Rodriguez-Grande et al., 2014; Rajkovic et al., 2018). Despite this, our recent study found that PTX3 mediates delayed neuroprotection after stroke, which is also supported by a previous study demonstrating that PTX3 KO mice have greater neuronal damage than WT mice in an N-methylD-aspartate-based epileptic seizure model (Ravizza et al., 2001). 

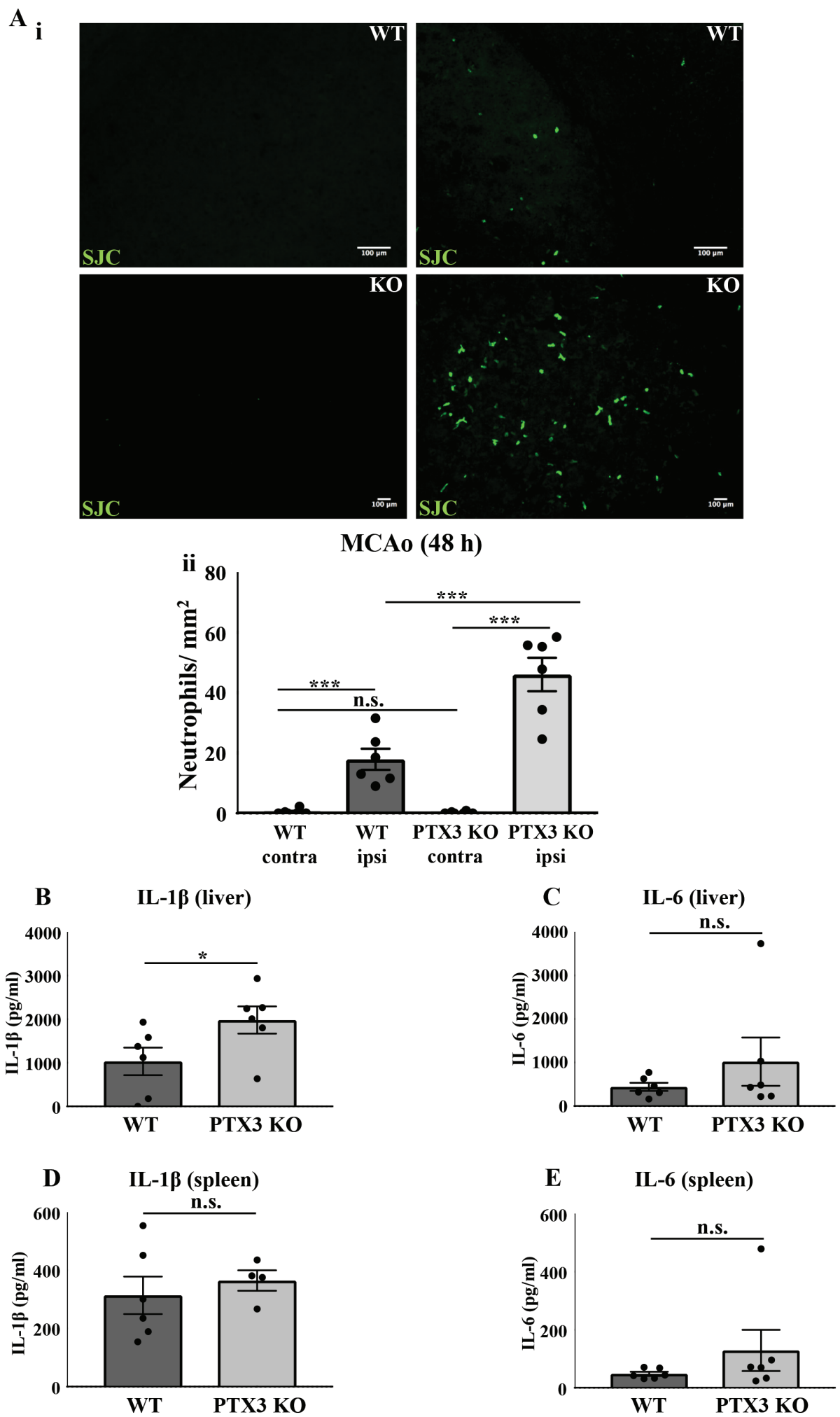

Figure 3. РТХ3 KO mice exhibit increased neutrophil infiltration into the brain $\mathbf{4 8} \mathbf{~ h}$ after cerebral ischaemia. (Ai) Neutrophils (green) labelled with SJC antibody in the contralateral and ipsilateral (ischaemic) area of the brain $48 \mathrm{~h}$ after stroke in WT and PTX3 KO mice. Scale bar, $100 \mu \mathrm{M}$. (Aii) The number of neutrophils per $\mathrm{mm}^{2}$ in contralateral and ipsilateral hemispheres of WT and PTX3 KO mice were quantified manually with ImageJ software. (B) IL-1 $\beta$ and (C) IL-6 levels $(\mathrm{pg} / \mathrm{ml})$ in liver and (D) IL-1 $\beta$ and (E) IL-6 levels in the spleen of WT and PTX3 $\mathrm{KO}$ mice were detected by ELISA. Statistical analyses were performed with repeated measures two-way ANOVA followed by Sidak corrected post-hoc analysis (ns $\left.\mathrm{P}>0.05,{ }^{* * *} \mathrm{P}<0.001\right)(\mathbf{A})$, and Student's t-test (ns $\left.\mathrm{P}>0.05,{ }^{*} \mathrm{P}<0.05\right)(\mathbf{B}-\mathbf{E})$. All data expressed as mean $\pm \mathrm{SEM}$ $(\mathrm{n}=6$ per group) $(\mathbf{A}-\mathbf{E})$. 
Our data taken together support the hypothesis that PTX3 is critical in the regulation of neutrophil infiltration in CNS inflammatory disease, reducing neutrophil trafficking to the brain after inflammatory insult or cerebral ischaemia, and that the neuroprotective effect of PTX3 could occur independently of PTX3-mediated neutrophil infiltration. However, our study also demonstrates that PTX3 has a completely opposite effect on neutrophil infiltration in vitro compared to in vivo inflammatory conditions, possibly involving different mechanisms due to discrepancies in the models used, further suggesting that any in vitro investigations must be validated in vivo. Our in vivo data, however, propose that PTX3 could be targeted therapeutically to reduce inflammatory-mediated damage and provide neuroprotection after cerebral ischaemia and potentially other cerebrovascular inflammatory diseases.

\section{Data availability}

Figshare: Pentraxin 3 regulates neutrophil infiltration to the brain during neuroinflammation. https://doi.org/10.6084/ m9.figshare.8088590 (Pinteaux, 2019).

This project contains Rajkovic_AMRC_Raw_Data.zip, which comprises underlying data stratified by the figure in which they appear.

Data are available under the terms of the Creative Commons Attribution 4.0 International license (CC-BY 4.0).

\section{Author contributions}

IR planned all of the experiments, performed the genotyping of animals, tissue collection and processing, histology, imaging, and data analysis, assisted with MCAo surgeries, and wrote the manuscript. RW conducted the MCAo surgeries. EL carried out the intrastriatal LPS injections. RT conducted the ELISAs for liver and spleen samples. EP and SA devised the study, obtained funding, and contributed to writing of the manuscript.

\section{Grant information}

This work was supported by funding from the British Heart Foundation (Reference: FS/14/70/31006), the Stroke Association (Reference: TSA2017/03) and the Medical Research Council (Reference: MR/N003586/10).

The funders had no role in study design, data collection and analysis, decision to publish, or preparation of the manuscript.

\section{Acknowledgements}

The authors would like to thank Dr. Cecilia Garlanda (Department of Immunology and Inflammation, Humanitas Clinical and Research Center, Italy) for providing PTX3 KO mice, and the Bioimaging Facility in the Faculty of Biology, Medicine and Health at the University of Manchester for equipment and advice in imaging. The anti-neutrophil primary antibody (SJC, rabbit anti-mouse, 1:10000) used in our study was kindly donated by Dr. Sandra J. Campbell, University of Cambridge, UK.
Allen C, Thornton P, Denes A, et al:: Neutrophil cerebrovascular transmigration triggers rapid neurotoxicity through release of proteases associated with decondensed DNA. J Immunol. 2012; 189(1): 381-392. PubMed Abstract | Publisher Full Text | Free Full Text

Bonacina F, Baragetti A, Catapano AL, et al:: Long pentraxin 3: experimental and clinical relevance in cardiovascular diseases. Mediators Inflamm. 2013; 2013 725102.

PubMed Abstract | Publisher Full Text | Free Full Text

Deban L, Russo RC, Sironi M, et al.: Regulation of leukocyte recruitment by the long pentraxin PTX3. Nat Immunol. 2010; 11(4): 328-334.

PubMed Abstract | Publisher Full Text

Erreni M, Manfredi AA, Garlanda C, et al:: The long pentraxin PTX3: A

prototypical sensor of tissue injury and a regulator of homeostasis. Immunol Rev. 2017; 280(1): 112-125.

PubMed Abstract | Publisher Full Text

Giles JA, Greenhalgh AD, Davies CL, et al:: Requirement for interleukin-1 to drive brain inflammation reveals tissue-specific mechanisms of innate immunity. Eur J Immunol. 2015; 45(2): 525-530.

PubMed Abstract | Publisher Full Text | Free Full Text

Jaillon S, Peri G, Delneste $Y$, et al:: The humoral pattern recognition receptor PTX3 is stored in neutrophil granules and localizes in extracellular traps. $J$ Exp Med. 2007; 204(4): 793-804.

PubMed Abstract | Publisher Full Text | Free Full Text

Jickling GC, Liu D, Ander BP, et al:: Targeting neutrophils in ischemic stroke: translational insights from experimental studies. J Cereb Blood Flow Metab. 2015; 35(6): 888-901.

PubMed Abstract | Publisher Full Text | Free Full Text

Kilkenny C, Browne WJ, Cuthill IC, et al:: Improving bioscience research reporting: the ARRIVE guidelines for reporting animal research. Osteoarthritis Cartilage. 2012; 20(4): 256-260.

PubMed Abstract | Publisher Full Text

Kim JY, Park J, Chang JY, et al.: Inflammation after Ischemic Stroke: The Role of Leukocytes and Glial Cells. Exp Neurobiol. 2016; 25(5): 241-251.

PubMed Abstract | Publisher Full Text | Free Full Text

Meeker RB, Williams K, Killebrew DA, et al.: Cell trafficking through the choroid

plexus. Cell Adh Migr. 2012; 6(5): 390-396.

PubMed Abstract | Publisher Full Text | Free Full Text
Pinteaux E: Pentraxin 3 regulates neutrophil infiltration to the brain during neuroinflammation. figshare. Dataset. 2019.

Rajkovic I, Wong R, Lemarchand E, et al:: Pentraxin 3 promotes long-term cerebral blood flow recovery, angiogenesis, and neuronal survival after stroke. J Mol Med (Berl). 2018; 96(12): 1319-1332.

PubMed Abstract | Publisher Full Text | Free Full Text

Ravizza T, Moneta D, Bottazzi B, et al.: Dynamic induction of the long pentraxin PTX3 in the CNS after limbic seizures: evidence for a protective role in seizureinduced neurodegeneration. Neuroscience. 2001; 105(1): 43-53.

PubMed Abstract | Publisher Full Text

Rodriguez-Grande B, Swana M, Nguyen L, et al.: The acute-phase protein PTX3 is an essential mediator of glial scar formation and resolution of brain edema after ischemic injury. J Cereb Blood Flow Metab. 2014; 34(3): 480-488. PubMed Abstract | Publisher Full Text | Free Full Text

Ryu WS, Kim CK, Kim BJ, et al:: Pentraxin 3: a novel and independent prognostic marker in ischemic stroke. Atherosclerosis. 2012; 220(2): 581-586. PubMed Abstract | Publisher Full Text

Salio M, Chimenti S, De Angelis N, et al:: Cardioprotective function of the long pentraxin PTX3 in acute myocardial infarction. Circulation. 2008; 117(8): 1055-1064. PubMed Abstract | Publisher Full Text

Schwartz M, Baruch $\mathrm{K}$ : The resolution of neuroinflammation in neurodegeneration: leukocyte recruitment via the choroid plexus. EMBO J. 2014; 33(1): 7-22

PubMed Abstract | Publisher Full Text | Free Full Text

Shindo A, Tanemura H, Yata K, et al.: Inflammatory biomarkers in atherosclerosis: pentraxin 3 can become a novel marker of plaque vulnerability. PLoS One. 2014; 9(6): e100045.

PubMed Abstract | Publisher Full Text | Free Full Text

Takeshita Y, Ransohoff RM: Inflammatory cell trafficking across the blood-brain barrier: chemokine regulation and in vitro models. Immunol Rev. 2012; 248(1): 228-239.

PubMed Abstract | Publisher Full Text | Free Full Text

Wong R, Lenart N, Hill L, et al:: Interleukin-1 mediates ischaemic brain injury via distinct actions on endothelial cells and cholinergic neurons. Brain Behav Immun. 2019; 76: 126-138.

PubMed Abstract | Publisher Full Text | Free Full Text 


\section{Open Peer Review}

\section{Current Peer Review Status:}

\section{Version 1}

Reviewer Report 02 July 2019

https://doi.org/10.21956/amrcopenres.13941.r26420

(C) 2019 Jaillon S. This is an open access peer review report distributed under the terms of the Creative Commons Attribution License, which permits unrestricted use, distribution, and reproduction in any medium, provided the original work is properly cited.

\section{Sébastien Jaillon}

Humanitas Clinical and Research Center, Humanitas University, Milan, Italy

In this manuscript, Rajkovic et al aimed to assess the role of PTX3 in neutrophil infiltration to the brain.

Authors showed increased recruitment of neutrophil in the brain of PTX3 KO mice, compared to wild type animals. The study is interesting and the manuscript well written.

However, the study has some limitations.

In particular, the discrepancy observed between in vitro and in vivo experiments remains unexplained.

Some specific questions:

Is it possible to modify the transmigration of neutrophils adding recombinant PTX3 in the system?

The authors did not take into account the production of PTX3 by the bEnd5 cells. Why? When stimulated with IL-1b, endothelial cells can produce PTX3.

In their in vivo models, authors should assess whether the levels of chemokines involved in recruitment of neutrophils are altered in PTX3 ko mice.

Is it possible to limit neutrophil infiltration in the brain by administrating recombinant PTX3?

Is the work clearly and accurately presented and does it cite the current literature? Yes

Is the study design appropriate and is the work technically sound? Yes

Are sufficient details of methods and analysis provided to allow replication by others? Yes 


\section{If applicable, is the statistical analysis and its interpretation appropriate?}

I cannot comment. A qualified statistician is required.

\section{Are all the source data underlying the results available to ensure full reproducibility? No source data required}

\section{Are the conclusions drawn adequately supported by the results? \\ Yes}

Competing Interests: No competing interests were disclosed.

Reviewer Expertise: Immunology

I confirm that I have read this submission and believe that I have an appropriate level of expertise to confirm that it is of an acceptable scientific standard.

Reviewer Report 29 May 2019

https://doi.org/10.21956/amrcopenres.13941.r26391

(C) 2019 Luheshi G et al. This is an open access peer review report distributed under the terms of the Creative Commons Attribution License, which permits unrestricted use, distribution, and reproduction in any medium, provided the original work is properly cited.

\section{Giamal N. Luheshi}

Department of Psychiatry, Douglas Mental Health University Institute, McGill University, Montreal, QC, Canada

\section{Argel Aguilar Valles}

Department of Neuroscience, Carleton University, Ottawa, Canada

The manuscript by Rajkovic et al investigated the role of Pentraxin 3 (PTX3) on neutrophil transmigration to the brain using in vitro and in vivo models. This is a very interesting study that builds on the authors substantial and innovative work on the role of neutrophils in the regulation of neuroinflammation, particularly in stroke models. The focus on PTX3 is novel and by investigating the role of this acute phase protein the authors hope to identify a suitable target for therapeutic intervention. The manuscript is very well written with very detailed methods and results section and the outcomes were discussed in the context of the wider field. There are a number of relatively minor general and more specific comments that the authors may want to address to further clarifying some aspects of the work:

1. Whilst the use of intracerebral injection of LPS is somewhat justified as a model of neuroinflammation, it is rather severe and importantly of little translational value, compared to the MCAO approach and even to systemic LPS which has been shown previously to trigger significant neutrophil transmigration through an IL-1b dependent manner.

2. It is surprising, given the history of this group, that little attention was paid to central cytokines as opposed to systemic ones measured in the in vivo studies of the present 
investigation. Particularly important would have been assessing the levels of not just the pro-inflammatory mediators but also the anti-inflammatory ones e.g.IL-1 ra (in which the authors have vast experience) and IL-10. Also, interesting would have been assessing the level some of the chemoattractant chemokines involved in the neutrophil transmigration process, especially in the KO animals. It would have been interesting to see if the levels of these mediators changed in the brain in line with the increased transmigration process that the authors reported in the $\mathrm{KO}$ animals.

3. The finding that the mechanism underlying the regulation of neutrophil infiltration by PTX3 is possibly independent from its reported neuroprotective effects is interesting. Did the authors consider testing this in neutropenic animals?

4. The In vitro and in vivo results are very clear, yet discrepant. Given the strength of the effects in the two systems, I believe is worth going further in the analysis of this discrepancy. For example:

Is it possible that survival of PTX3 KO neutrophils is compromised in a wildtype milieu?

Are PTX3 KO endothelial cells expressing something that promotes transmigration (which is obviously not present in the in vitro system)?

What would happen to WT or PTX3KO neutrophil transmigrating through PTX3 KO endothelial cells?

It is understandable why the authors used the in vitro approach to investigate their hypothesis, however given the different results in the two systems this rather distracts from the most interesting findings in vivo. I think a stronger justification for including these results and more indepth discussion, other than stating...possibly involving different mechanism.. is warranted here.

Is the work clearly and accurately presented and does it cite the current literature? Yes

Is the study design appropriate and is the work technically sound? Yes

Are sufficient details of methods and analysis provided to allow replication by others? Yes

If applicable, is the statistical analysis and its interpretation appropriate? Yes

Are all the source data underlying the results available to ensure full reproducibility? Yes

Are the conclusions drawn adequately supported by the results? Yes

Competing Interests: No competing interests were disclosed.

Reviewer Expertise: Neuroscience, neuroinflammation, sickness behaviour

We confirm that we have read this submission and believe that we have an appropriate level of expertise to confirm that it is of an acceptable scientific standard. 